\title{
Solar conversion
}

\section{Non-aqueous electrolytes make wet cells work}

from S. Dennison

IN the urgent search for photovoltaic devices for the direct conversion of solar energy to electricity, solid-state solar cells have in the past few years far outstripped their counterparts based on junctions between semiconductors and electrolytes. Although 'wet' photovoltaic cells may be more easily manufactured, they are far behind as practical converters. But now there is a prospect that this gap may be closed.

At the end of 1982, Stanford University chemists Chris Gronet and Nathan Lewis described (Nature 300, 733; 1982) a cell based on an $\mathrm{n}-\mathrm{GaAs}_{1-x} \mathrm{P}_{\boldsymbol{X}}$ semiconductor in contact with a non-aqueous redox electrolyte with an efficiency of no less than 13 per cent, a doubling of the best previous efficiency. The problems that had plagued earlier devices were overcome by optimizing the semiconductor properties and preventing corrosion by the use of a non-aqueous electrolyte. Now, Gronet and Lewis, together with C. Lieber, have made an equally impressive step forwards by the construction of a 10 per cent efficient cell based on the rather more familiar material p-type silicon, in a non-aqueous electrolyte containing the cobaltocene-cobaltocinium redox couple (see this issue of Nature, p.533).

The efficiency of 10 per cent, obtained both with a laboratory lamp and direct sunlight, dwarfs that of previous cells based on p-silicon, putting it high in any league table of 'wet' photovoltaic cells. At the same time, the Stanford group has demonstrated a major departure from the expected behaviour of p-type silicon in contact with a non-aqueous electrolyte and have shown how certain intrinsic limitations on cell performance expected from the behaviour of the semiconductorelectrolyte interface are not apparent in their cell.

To a large extent, the major problems of the liquid-junction photovoltaic cell (see our discussion, Nature 300,$687 ; 1982$ ) have been solved by the replacement of aqueous with non-aqueous electrolytes. By the rigorous exclusion of water, semiconductor corrosion is almost completely suppressed. These solvents also allow a far wider range of redox couples to be used, so that difficulties of poor electron-transfer kinetics can be overcome. But the use of non-aqueous media introduced a further complication - an apparent limitation of the photovoltage obtainable from a cell, a phenomenon now known as 'Fermi level pinning'.

The phenomenon is this. With a number of semiconducting materials, the observed photovoltage seems to be more or less

constant for each material and independent of the redox couples in solution with which it may be in contact. But on the conventional model of such an interface, the photovoltage should reflect the difference between the redox potential in solution and the Fermi level of the semiconductor. The observation that the photovoltage is substantially constant thus implies a constant difference between the redox potential and the Fermi level, which in turn suggests that the redox couples in the non-aqueous medium are in some way 'pinning' the Fermi level.

The explanation lies in the charge distribution at the interface. On the conventional view, there should be a depletion layer beneath the surface of the semiconductor across which most of the potential difference between the bulk semiconductor and the bulk electrotype should be dropped. A change in the redox potential should then alter the potential gradient within the depletion layer and thus the photovoltage. But if there are electronic states at the semiconductor surface which can be charged and discharged, the potential gradient at the surface will be substantially different from that expected and the interface more like the junction between a metal and an electrolyte. Such surface states may arise from bulk defects in the crystal lattice, from dangling bonds at the surface or by the adsorption of charged species onto the

\section{Molecular biology}

\section{Selfish DNAs with self-restraint}

\author{
from W.F. Doolittle, T.B.L. Kirkwood and M.A.H. Dempster
}

TRANSPOSABLE elements are small discrete genetic elements which can be replicated independently of the chromosomes in which they are embedded. Mechanisms of independent replication may vary, but the results are almost always the same - new copies appear at new genomic positions without loss of original copies' . Models of how transposition could occur have been with us for some time and now we are beginning to see models of how the process could be regulated.

In a recent issue of Cell, Kleckner and her colleagues describe the inwardly and outwardly directed promoters of IS10Right, one of the two copies of the insertion sequence IS10 which form the terminal repeats of the bacterial transposon $T n 10$ (refs 2,3). Transcription of the IS10encoded transposase gene initiates at the surface. The overall result is a characteristic photovoltage for each junction between a semiconductor and an electrolyte which is independent of the redox couple. It has recently been thought that behaviour of this type is common among small band-gap semiconductors, precisely those which are of most use for solar conversion, p-type silicon in particular. So Fermi pinning and the consequent loss of photoconversion efficiency has been regarded as the last nail in the coffin for liquid-junction photovoltaic devices.

The latest results from the Stanford group are particularly important because they show that device performance is not necessarily limited by the intrinsic properties of the interface between $\mathrm{p}-\mathrm{Si}$ and the non-aqueous electrolyte. The explanation is not yet clear, but it seems likely that there is an obverse phenomenon to 'Fermi pinning' which might be called 'redox-pinning'. In a photovoltaic device of the type described, both oxidized and reduced forms of the couple would accumulate at the surface. If the components of the redox couple can communicate with the bulk of the semiconductor much faster than the surface states, the filling of these states will be effectively determined by the relative concentrations of the components of the redox couple in solution. And if these vary little with the degree of bias on the semiconductor, then the junction will behave as a classical device.

It now remains to be seen whether these important findings can be translated into a working cell configuration without loss of conversion efficiency.

S. Dennison is at the Inorganic Chemistry Laboratory, University of Oxford, Oxford OXI $3 Q R$. weak inward promoter pIN. The stronger pOUT is responsible for activation of genes distal to the site of IS10 or Tn10 insertion. The two are transcriptionally independent, but active pOUTs inhibit, in trans, the expression of transposase genes. This translational inhibition is mediated, Simons and Kleckner ${ }^{3}$ suggest, "by direct pairing between the transposase messenger RNA and a small, complementary, regulatory RNA specified by the IS10encoded pOUT promoter".

This molecular model makes excellent sense of the genetic phenomenon of multicopy inhibition - the suppression of the transposition-related functions of a single $T n 10$ transposon by multiple copies of its IS10 terminal repeats elsewhere in the cell. It also makes evolutionary sense. Transposable elements are viewed by some as 